\title{
Marine controlled-source electromagnetic sounding on submarine massive sulphides using 2.5-D simulation
}

\author{
Naoto Imamura ${ }^{1}$, Tada-nori Goto ${ }^{1}$, Junichi Takekawa ${ }^{1}$ and Hitoshi Mikada ${ }^{1}$ \\ ${ }^{1}$ Dept. of Civil and Earth Res. Eng., Kyoto University
}

\begin{abstract}
Recently, controlled-source electromagnetic method is widely used for shallow sub-seafloor explorations. In this paper, we propose a new controlled-source electromagnetic method using two autonomous underwater vehicles (AUVs) for the exploration of submarine massive sulphides. A numerical simulation code for 2.5-D electromagnetic field in the frequency domain is developed in order to estimate electromagnetic responses on possible conductivity structures. As a result, we confirmed that the magnetic field on the seafloor is very sensitive to the horizontal distribution of buried conductive zone. We also confirmed that the intensity of received electric field decreases near the seafloor in an area wider than the horizontal extent of the buried conductive zone, when the current source dipole is located above the conductive zone. This is because electric field is attenuated due to the return current in the conductive zone. Based on our result, we think that the new controlled-source electromagnetic method is realizable.
\end{abstract}

\section{INTRODUCTION}

Recently, controlled-source electromagnetic (CSEM) method is widely used for shallow subsurface exploration to measure resistivity structure in detail. This method is also used for surveying oil and natural gas resources in deep sea $^{1), 6}$. These days, theoretical and experimental attempts have been initiated to survey submarine massive sulfides (SMS) using electromagnetic method. Kowalcyk ${ }^{4)}$ surveyed submarine massive sulphides using EM method with magnetic source, although the sounding depth will be limited within several meters.

In conventional marine CSEM methods, we need to connect a survey vehicle and an EM transmitter using a long cable, and also connect the EM transmitter and towed receiver using a cable. However, in practice, we must tow cables far from seafloor because of rough topography (e.g, chimneys) around submarine massive sulphides. Therefore, it is difficult to get information about shallow sub-seafloor structure. In this research, we propose a new marine CSEM method to solve this problem using two autonomous underwater vehicles (AUV). We set an EM transmitter to one AUV, and also set an EM receiver to another one. Using this method, it is possible to keep a low height of diving AUVs from the seafloor, so we can carry out the exploration of submarine massive sulphides effectively.

In CSEM method, the behavior of electric and magnetic fields are determined by how the dipoles are arranged against to the survey target. Therefore, it is important to consider the effective arrangement of transmitter and receiver in case of the structure including low resistivity anomaly. Moreover, we need to include the location of each source electrodes to the numerical modeling because the source-receiver separation will be quite shorter than the conventional CSEM survey.

In this research, we developed a new numerical program, and discussed the feasibility of the new marine CSEM method which uses two AUVs, considering real deep-sea exploration.

\section{METHOD}

In this paper, we employed a 2.5-dimentional (2.5D) simulation to deal a two dimensional (2-D) structure. The 2.5-D simulation is a numerical calculation assuming 3-D EM field from artificial source over 2-D earth. The 2.5-D simulation is a useful method when the behavior of earth structure is similar to 2-D like active fault or across structure. In order to simulate 2-D earth model ( $\mathrm{x}-\mathrm{z}$ plane) and 3-D electric dipole source (x-y-z space), we must apply the Fourier transform to y-component of electric (E) and magnetic field (H) and source terms. Then, we calculate the Maxwell equation 2$\mathrm{D}$ and wave domain ${ }^{3)}$. Assuming a time-dependence of $e^{i \omega t}$, the electric field $\mathbf{E}$ and magnetic field $\mathbf{H}$ are described by Maxwell's equations:

$$
\nabla \times \mathbf{E}=-\hat{z} \mathbf{H}-\hat{z} \mathbf{M}_{s}
$$

and 


$$
\nabla \times \mathbf{H}=\hat{y} \mathbf{E}+\mathbf{J}_{s}
$$

where $\mathbf{M}_{\mathbf{s}}$ and $\mathbf{J}_{\mathbf{s}}$ are the impressed magnetization and electric currents, respectively, and the impedivity $\hat{z}=i \mu \omega$ and the admittivity $\hat{y}=\sigma+i \varepsilon \omega^{5)}$ are used. Applying the Fourier transform to each component of $\mathbf{E}$ and $\mathbf{H}$ and source terms of equation (1) and (2) with respect to $y$ as

$$
\hat{\mathbf{F}}\left(x, k_{y}, z, \omega\right)=\int_{-\infty}^{\infty} \mathbf{F}(x, y, z, \omega) e^{-i k_{y} y} d y,(3)
$$

we obtain two coupled governing differential equations for $\hat{E}_{y}$ and $\hat{H}_{y}$. After applying finite element method, we obtain the linear equation. When we solve this linear equation and employ the inverse Fourier transform, the 3-D electric field in the model is derived, finally.

Before numerical experiments, we evaluated accuracy of our program. We compared the electric fields with EM1D, which is a 1-D CSEM numerical simulation program. The model considered in this comparison is a simple homogeneous model with no anomaly. The resistivity is set as $0.3 \Omega$ assuming sea resistivity. The parameter of source is determined same as the dipole strength in EM1D; $\mathrm{I}=1 \mathrm{~A}, \mathrm{dl}=1 \mathrm{~m}$. The frequency is also set as $10 \mathrm{~Hz}$. The comparison between our program and EM1D is shown in figure 1 .

The electric field is different within $60 \mathrm{~m}$ from transmitter. This difference probably comes from the limitation of numerical calculation of the inverse Fourier transform. The assumed grid spacing restricts stability condition, and gives us a limitation of y-component calculation with a large wave number. A short source-receiver separation requires larger wave numbers for the accurate inverse Fourier transform. That is why the calculated result and EM1D are different near the transmitter. Considering above conditions, we can calculate the electric field almost similar to EM1D from $60 \mathrm{~m}$ to $350 \mathrm{~m}$.

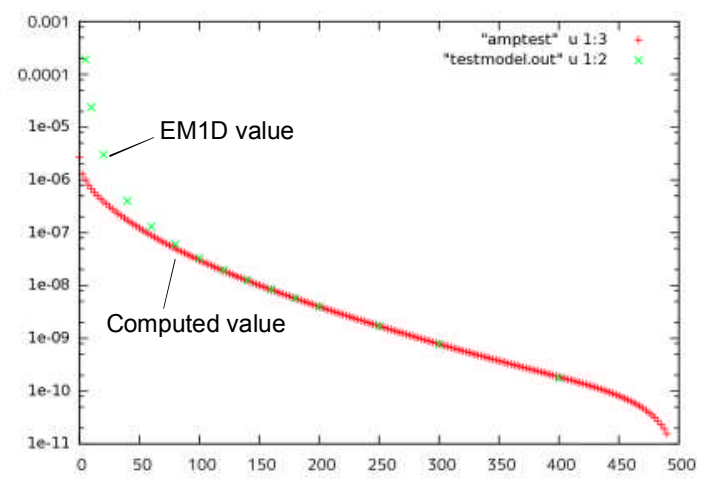

Figure 1. Accuracy of the program comparing EM1D

\section{MODEL}

Many explorations were made to survey the submarine massive sulphides near Japan or around the world (e.g., Kowalczyk, 2008), but we cannot get the detail cross section of submarine massive sulphides yet. We assume the submarine massive sulphides and chimney mound to be a simple rectangular block. We suppose the SMS's width to be $100 \mathrm{~m}$ and the thickness to be $20 \mathrm{~m}$, referring a structure of a hydrothermal mound ${ }^{2}$. We also suppose the chimney mound's width to be $20 \mathrm{~m}$ and change the height. The boundary condition is assumed that the electric and magnetic fields are equal to be zero in the far area. We consider that the representative models of submarine massive sulphides like figure 2 and chimney mound like figure 3. In figure 2 model, we change the depth of the SMS. We arranged the transmitter above the anomaly, and fixed the source amplitude as $1 \mathrm{~A}$ and the frequency as $10 \mathrm{~Hz}$. We also fixed the dipole length as $1 \mathrm{~m}$ which can be possible to carry with AUVs in practice. In figure 3 model, we change the height of the chimney mound. We arranged the receiver over the chimney mound and the source amplitude and the frequency same as figure 2

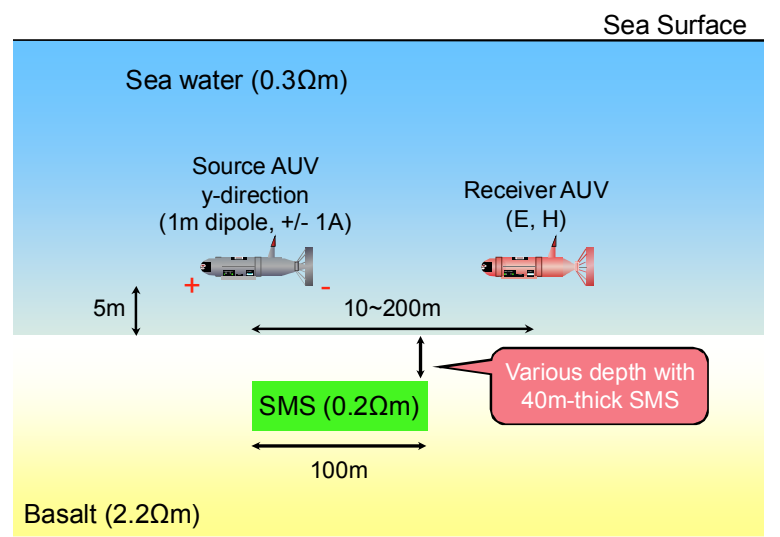

Figure 2. The model including the SMS

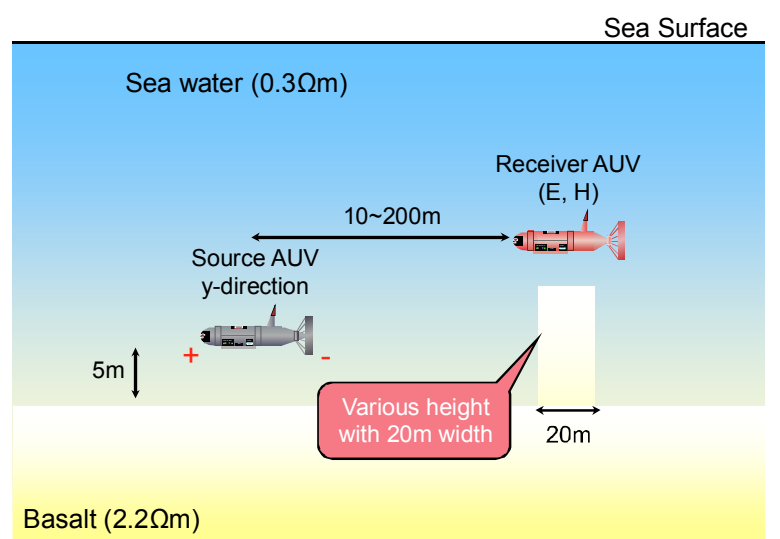

Figure 3. The model including the chimney mound 
model. We changed the distance between the source dipole and receiver dipole, and then compared the received electric amplitude. Before these numerical simulations, we calculated the electric amplitude of receiver noise per ampere. We calculated and compared this receiver noise assuming the electric current of transmitter to $50 \mathrm{~A}$, the dipole length of receiver to $4 \mathrm{~m}$, the electric amplitude of receiver noise to $10 \mu \mathrm{V}$. As a result, the predicted noise level of electric field is $5 \times 10^{-9} \mathrm{~V} / \mathrm{m}$ and magnetic field is $2 \times 10^{-6} \mathrm{~A} / \mathrm{m}$.

\section{RESULTS AND EXAMINATION}

We show the numerical results for figure 2 model from figure 4 to figure 6 and for figure 3 on figure 7 in the final page. In each figure, the $\mathrm{x}$-axis means the distance from source to receiver, and the y-axis means the amplitude of the calculated electric field and magnetic field or the abnormal amplitude. These figures show that received electric and magnetic field using CSEM method is greater than the noise level on specific range, so it is possible to observe the electric and magnetic field using the "AUV-CSEM" method in practice. For example, figure 4 shows that the received magnetic field is 300 percent larger than the non-anomaly case. Figure 4 also shows that the received magnetic field suddenly changes around the edge of the anomaly in case of the SMS's depth is zero. Figure 5 shows that the received magnetic field is 40 percent smaller than the non-anomaly case around the SMS's area. Figure 5 also shows that the abnormal amplitude doesn't change entirely on non-anomaly area. Figure 6 shows that the received electric field is 40 percent smaller than the non-anomaly case. Figure 6 also shows that the abnormal amplitude is still attenuating over the area of SMS. Figure 7 shows that the received magnetic field is greater than the non-anomaly case. Figure 7 also shows that the abnormal amplitude depends on the height of the chimney mound.

These behaviors are different from the case that the anomaly is high resistivity like oil or natural gas. When the earth structure includes high resistivity, the received electric field becomes asymptotic to the non-anomaly case at the observing point far from the boundary of the anomaly. However, when the earth includes conductivity such as the submarine massive sulphides, the received electric field doesn't become asymptotic to the nonanomaly case and keeps lower amplitude compared to the non-anomaly case. We consider that the conductive anomaly under transmitter make a short circuit of electric current near the source dipole, so the observed electric field becomes lower entirely.

\section{CONCLUSIONS}

In this research, we compared the behavior of electric and magnetic fields various a function of the distance between source and receiver, and discussed the possibility of our CSEM method to be applied for the exploration of submarine massive sulphides. From the simulation results, we found that it is possible to detect the electric and magnetic field for about $150 \sim 170 \mathrm{~m}$ offsets even under the contamination of noise. Among various combination of source and receivers, we also found that the anomalous amplitude rate becomes greatest, in particular around the edge of submarine massive sulphides, when polarizing the receiver in the $\mathrm{x}$ direction. We next considered the sensitivity of electromagnetic field to the location of submarine massive sulphides. We found that the received electric field becomes steeply weaker as setting the receiver apart from the transmitter when the source was placed near submarine massive sulphides.

Above all numerical calculations, we propose the new CSEM method using AUVs. We consider that it is possible to observe the electric and magnetic field within $150 \mathrm{~m}$ from the source with two AUVs. When the source-AUV is arranged near the anomaly, the received electric field by the other AUV would strongly be diminished in far offset. When the receiver AUV approaches near the boundary of submarine massive sulphides, the received magnetic field (x-comp) would show abrupt change near the horizontal boundary of the anomaly.

In near future, we plan to analyze $\mathrm{x}$-direction and $\mathrm{z}$ direction transmitter and compare the amplitude of electric and magnetic field with anomaly or without anomaly. We also plan to make a complex earth model including complicated shape of submarine massive sulphides and complex topography around the hydrothermal area.

\section{ACKNOWLEDGMENTS}

We are thankful for Dr. Ki ha Lee for allowing us to use EM1D for simulation of 1-D CSEM cases. We also thank for comments and suggestions from Dr. Noriko Tada and Dr. Takafumi Kasaya in JAMSTEC.

\section{REFERENCES}

1) Constable, S., and Srnka, L. J. (2007): An introduction to marine controlled-source electromagnetic methods for hydrocarbon exploration. Geophysics, 72, WA3-WA1

2) Humphris, S. E. et al. (1995): The internal 
structure of an active sea-floor massive sulphide deposits: Nature 377, 713-716

3) Mitsuhata, Y.,Uchida,T., and Amanoz, H., (2002) : 2.5-D inversion of frequency-domain electromagnetic data generated by a groundedwire source,Geophysics, 67,6,1753-1768

4) Peter Kowalczyk (2008): Geophysical prelude to first exploitation of submarine massive sulphides, first break 26, November 2008, 99106

5) Ward, S. H., and Hohmann, G. W., 1988, Electromagnetic theory for geophysical applications, in Nabighian, M. N., Ed., Electromagnetic methods in applied geophysics, 1, Theory: Soc. Explor. Geophys.

6) Yamane, K. (2008): Possibility of application of marine CSEM sounding method to oil/gas explorations, JOGMEC review of Oil and Gas, 42, 55-73 (in Japanese) 

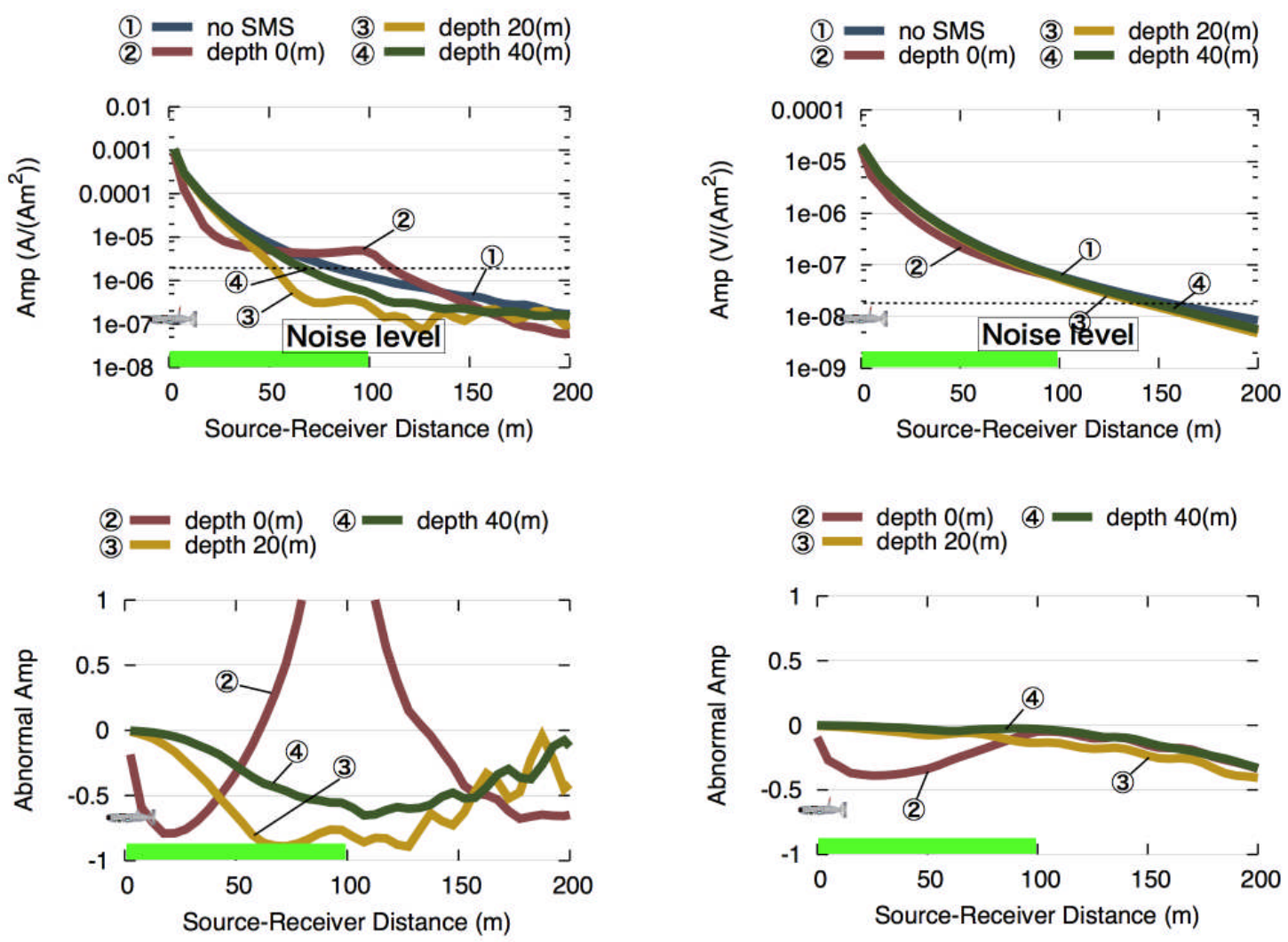

Figure 4. Amplitude of the magnetic field and the anomaly with the distance between source and receivers. The receiver toward $\mathrm{x}$ direction is arranged on the model in figure 2.
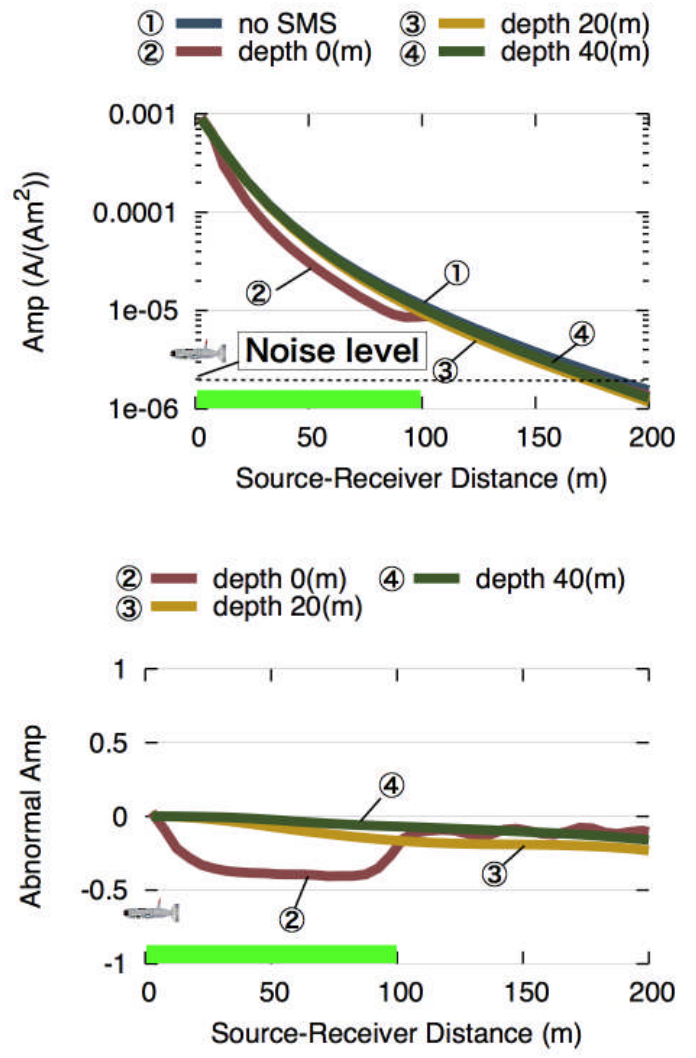

Figure 5. Amplitude of the magnetic field and the anomaly with the distance between source and receivers. The receiver toward $\mathrm{z}$ direction is arranged on the model in figure 2 .

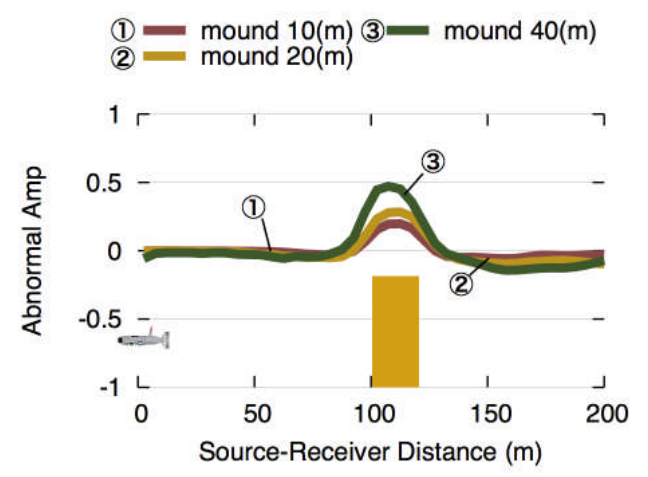

Figure 7. Amplitude of the magnetic field and the anomaly with the distance between source and receivers. The receiver toward $\mathrm{z}$ direction is arranged on the model in figure 3. 